\title{
Getting old and cancer: hand-in-hand?
}

DOI:

10.1038/nrc2215
The p53-ARF pathway is known to suppress tumorigenesis. p53 is activated in response to high levels of DNA damage (which frequently occur in cancer cells) and it has also been suggested that p53 responds to chronic low levels of DNA damage (which are associated with ageing) to induce senescence or apoptosis. ARF is activated in response to oncogenic signalling and the cellular effects of ageing and stabilizes p53; thus, when p53 is activated, ARF amplifies the activity of p53. Manuel Serrano and colleagues now provide evidence that the p53-ARF pathway increases cellular resistance to oxidative damage in mice, which protects against ageing.

Previously, mice that expressed a single additional copy of $\operatorname{Tr} p 53$ (encoding p53) or Cdkn2a (encoding ARF) - termed super-p53 (s-p53) and s-ARF mice, respectively - were shown to have a significantly reduced incidence of cancer, although the mice aged normally. To investigate whether the concomitant expression of both tumour suppressors had a synergistic effect, the authors crossed the s-p53 and s-ARF mice to generate s-p53;s-ARF mice. Crucially, in mouse embryonic fibroblasts (MEFs) derived

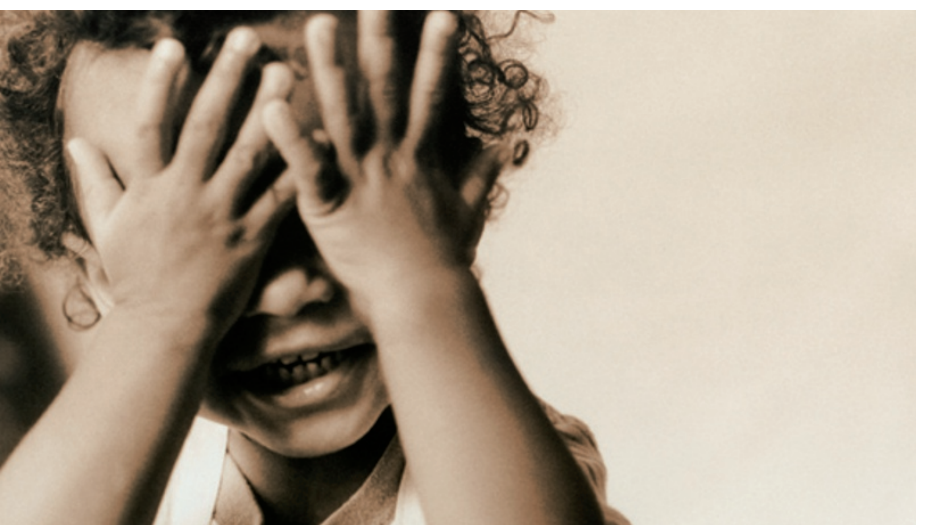

from s-p53;s-ARF mice, p53 and $\mathrm{ARF}$ were regulated normally, despite exhibiting increased expression on activation. Primary MEFs exhibited a marked resistance to immortalization and were resistant to transformation. Consistently, aged s-p53;s-ARF mice had a significantly reduced spontaneous tumour incidence and showed delayed development of chemically induced fibrosarcomas and papillomas. They also showed that s-p53; s-ARF mice have a significantly longer average lifespan than their wild-type and single-transgene-expressing counterparts. However, the maximum lifespan of the s-p53;s-ARF mice was similar to that of wild-type mice, indicating that p53 and ARF function synergistically to reduce some, but not all, of the underlying causes that limit longevity.

To assess whether the reduced susceptibility to cancer might account for the delayed ageing in sp53;s-ARF mice, the authors showed that aged, cancer-free s-p53;s-ARF mice had an extended average lifespan compared with wild-type mice, but that there was no difference in the incidence of ageing-associated diseases, such as benign growths. By contrast, hallmarks of ageing were significantly reduced in the s-p53;s-ARF mice - neuromuscular coordination and hair regrowth were significantly improved and levels of phosphorylated histone $\mathrm{H} 2 \mathrm{AX}$ (a marker of DNA damage that accumulates in ageing cells) were reduced in vivo. Together, these data indicate that p53 and ARF cooperate to protect cells from ageing and that the improved survival of the s-p53;sARF mice might not be the result of increased resistance to tumorigenesis.
How do p53 and ARF confer this protection? Although the authors note that telomere length was slightly increased, they showed that both young and aged s-p53;s-ARF mice exhibited a marked reduction in the levels of reactive oxygen species, which cause oxidative damage to DNA, lipids and proteins and contribute to the chronic damage that is associated with the ageing process. Moreover, oxidized lipids and proteins were reduced in tissues from both young and old mice, indicating that p53 and ARF protect against oxidative damage throughout the lifespan of the mice. Two targets of the antioxidant transcriptional programme induced by p 53 stabilization, sestrin-1 (Sesn1) and Sesn2, were highly expressed in vitro and in vivo, and s-p53;s-ARF mice exhibited increased resistance to lethal doses of the oxidative agent paraquat, providing further evidence that the s-p53;s-ARF mice have an elevated antioxidant response.

Previous studies indicated that p53 activation results in premature ageing; however, Serrano and colleagues speculate that these contrasting observations could be explained by the constitutive expression of activated p53 in the earlier studies, whereas p53 is regulated normally in s-p53;s-ARF mice. Therefore, these data indicate that heightened protection from oxidative damage, in this case owing to increased p53- and ARF-dependent responses, delays the ageing process and that this might be coupled with reduced susceptibility to cancer.

Gemma Alderton

ORIGINAL RESEARCH PAPER Matheu, A. et al. Delayed ageing through damage protection by the Arf/p53 pathway. Nature 448, 375-379 (2007) 